\title{
5 BUSCANDO INFORMAÇÕES EM SAÚDE ONLINE: ESTRATÉGIA DE ENFRENTAMENTO DOS ADOLESCENTES COM DOENÇAS CRÔNICAS1
}

\author{
| Gabriela dos Santos²; Cláudia Tavares ${ }^{3}$; Rosane de Aguiarr; Ana Queiroz ${ }^{5}$; Rejane Ferreira ${ }^{6}$; Cosme Pereira ${ }^{7}$ |
}

\section{RESUMO}

CONTEXTO: Com a internet e as redes virtuais, é possível encontrar informações a respeito de diversos assuntos, de acordo com o interesse do público, e ainda criar grupos para discussões sobre os temas.

OBJETIVO: Conhecer os aspetos positivos e negativos relacionados à busca de informações em saúde na internet por adolescentes com doença crônica.

METODOLOGIA: Pesquisa qualitativa, realizada a partir de entrevistas semiestruturadas com adolescentes que se tratam no ambulatório de um hospital universitário no Rio de Janeiro.

RESULTADOS: Foram apontados aspetos positivos como a compreensão da doença crônica e a influência da mídia no tratamento e no autocuidado. Além disso, aspetos negativos como a ausência de conteúdos inteligíveis e a dificuldade para identificar a segurança do site e veracidade das informações foram apresentadas.

CONCLUSÕES: Isso nos mostra que a presença do enfermeiro no ambiente virtual deve ser valorizada como marco orientador e produtor de informações idôneas sobre saúde e cuidado.

PALAVRAS-CHAVE: Adolescente; Doença crônica; Rede social

\section{RESUMEN}

"Buscando información de salud en línea: Estrategias de enfrentamiento de los adolescentes con enfermedades crónicas"

CONTEXTO: Con el internet y las redes virtuales, es posible encontrar información acerca de diversos temas, de acuerdo con el interés del público, y también crear grupos para discusiones sobre los tópicos.

OBJETIVO: Conocer los aspectos positivos y negativos relacionados con la búsqueda de información en salud en el internet por parte de los adolescentes con enfermedad crónica.

METODOLOGÍA: Investigación cualitativa, efectuada a partir de entrevistas semiestructuradas con adolescentes tratados en la unidad ambulatoria de un hospital universitario de Rio de Janeiro. RESULTADOS: Se han indicado aspectos positivos como la comprensión de la enfermedad crónica y la influencia de los medios de comunicación en el tratamiento y el autocuidado. Además, se presentaron aspectos negativos como la falta de contenidos inteligibles y la dificultad para identificar la seguridad del sitio web y la veracidad de la información.

CONCLUSIONES: Esto nos pone de manifesto que la presencia del enfermero en el entorno virtual debe ser valorada como un marco orientador y productor de información idónea sobre la salud y los cuidados de salud.

\section{DESCRIPTORES: Adolescente; Enfermedad crónica; Red social}

\section{ABSTRACT \\ "Pursuing online health information: Coping strategies of ado- lescents with chronic diseases"}

BACKGROUND: The Internet and the virtual network allow us to locate information regarding several different matters according to the public interest, and also to create groups for discussions about these.

AIM: To understand the positive and negative aspects related to the pursuit of health information on the internet by adolescents with chronic disease.

METHODS: Qualitative research, held from semi-structured interviews with adolescents undergoing treatment in the outpatient unit of a university hospital in Rio de Janeiro.

RESULTS: Positive aspects such as the understanding of the chronic disease and the influence of media both in the treatment and the self-care procedures have been pinpointed. Furthermore, negative aspects such as the lack of intelligible contents and the difficulty in identifying the safety of the websites as well as the veracity of the information were presented.

CONCLUSIONS: This proves to us that the presence of the nursing professional in the virtual environment should be valued as a benchmark that guides and produces trustworthy information about health and care.

\section{KEYWORDS: Adolescent; Chronic disease; Social network}

Submetido em 20-12-2015

Aceite em 30-05-2016

\footnotetext{
1 Artigo extraído da Dissertação de Mestrado intitulada "Busca de informação em saúde nas redes sociais virtuais por adolescentes com doenças crônicas: Contribuição da enfermagem”, apresentado no I Encontro Internacional Inovação no Ensino na Saúde e Enfermagem.

2 Enfermeira; Doutoranda na Universidade Federal do Rio de Janeiro, Escola de Enfermagem Anna Nery, Cidade Nova, Rio de Janeiro, Brasil, sisan.gabi@hotmail.com 3 Enfermeira; Professora Titular na Universidade Federal Fluminense, Escola de Enfermagem Aurora de Afonso Costa, Niterói - RJ, Brasil, claudiamarauff@gmail.com 4 Enfermeira; Professora na Universidade Federal Fluminense, Escola de Enfermagem Aurora de Afonso Costa, 24020-091 Niterói - RJ, Brasil, rcburla@yahoo.com.br 5 Enfermeira; Professora Associada na Universidade Federal do Rio de Janeiro, Escola de Enfermagem Anna Nery, Rio de Janeiro - RJ, Brasil, abaqueiroz@hotmail.com 6 Enfermeira; Doutoranda na Universidade Federal Fluminense, Escola de Enfermagem Aurora de Afonso Costa, Niterói - RJ, Brasil, rejane_eleuterio@hotmail.com 7 Enfermeira; Mestre pela Universidade Federal Fluminense, Escola de Enfermagem Aurora de Afonso Costa, 24020-091 Niterói - RJ, Brasil, cosmehesfa@yahoo.com.br
} 


\section{INTRODUÇÃO}

As redes sociais virtuais têm inúmeras vantagens e uma delas é a facilidade de acesso em qualquer ponto onde a internet esteja disponível para a conexão. Em todas elas são possíveis encontrar e compartilhar informações a respeito de diversos assuntos, de acordo com o interesse do público, e ainda criar grupos para discussões específicas. Esse recurso beneficia pessoas confinadas a um determinado espaço, impossibilitadas, total ou parcialmente, de estabelecer contato com outros grupos sociais - seja com um membro familiar, amigos e até mesmo com profissionais da saúde.

Analisando a rede virtual, podemos apontá-la como um amplo campo através da qual descobrimos novos recursos e ferramentas para divulgação e busca de informações sobre doenças físicas e psicológicas, em um curto intervalo de tempo e com acesso local, regional, nacional e internacional. Podemos considerar que as mídias estão presentes na vida das pessoas e o crescente número de blogs e portais para pacientes mostra que muitos estão ativos nessas redes virtuais (Velden \& Khaled, 2013). Greene, Choudhry, Kilabuk \& Shrank (2011) mostram que o facebook tem sido um ponto importante de encontro online, com mais de 400 milhões de usuários registrados em todo o mundo. Nele, muitos sites de grupos sobre doença, surgiram representando fontes de informação, apoio para pacientes com doenças crônicas, entre esses encontramos os adolescentes (Greene et al, 2011).

A adolescência constitui-se como uma fase de vida crítica para a saúde mental e são várias as perturbações mentais que têm o seu pico de incidência nestas fases, nomeadamente a depressão e tentativas de suicídio, uso de substâncias, perturbações alimentares, ansiedade e psicoses (Morgado \& Botelho, 2014). Esses conflitos internos se potencializam quando o adolescente possui em seu histórico uma doença crônica.

Com a internet e as mídias, inúmeras redes virtuais têm-se tornado ferramentas de grande utilidade tanto para os profissionais de saúde quanto para as pessoas envolvidas no processo saúde e doença do adolescente, o que contribui de forma positiva para o enfrentamento da doença crônica, seja por meio dos conteúdos disponibilizados relacionados à saúde ou de bate-papo (Santos, Tavares, Ferreira \& Pereira, 2015).

Assim, este estudo buscou conhecer os aspetos positivos e negativos relacionados à busca de informações sobre doença crônica por adolescentes nas redes sociais virtuais.

\section{METODOLOGIA}

Trata-se de estudo descritivo-exploratório de abordagem qualitativa, realizado com adolescentes, que estão em tratamento de patologias crônicas como Lúpus Eritematoso Sistêmico, Doença Renal Crônica, Câncer e Insuficiência pulmonar, no Núcleo de Estudos da Saúde do Adolescente (NESA) Do Hospital Universitário Pedro Ernesto (HUPE), da Universidade do Estado do Rio de Janeiro/ Brasil. A partir de um convite formal, tornaram-se participantes deste estudo 14 adolescentes, que em algum momento buscaram informações sobre saúde nas redes virtuais. A coleta de dados se deu através de entrevistas semiestruturadas, individuais, no período de junho a setembro de 2014, nos dias de atendimentos estabelecidos pelo ambulatório de especialidades do NESA. Os critérios de exclusão adotados foram os seguintes: pacientes que nunca acessaram quaisquer mídias virtuais para obtenção de informações relacionadas à saúde a fim de compreender o processo da doença crônica, adolescentes com défice cognitivo e analfabetos e menores de 18 anos cujos responsáveis não consentiram a participação na pesquisa.

As questões que nortearam a entrevista foram: Como você sabe que o conteúdo encontrado na internet é confiável? Os conteúdos encontrados no ambiente virtual têm-lhe ajudado com seus problemas de saúde? O que você já sabe sobre as formas de lidar com seus problemas de saúde?

As entrevistas foram gravadas com o assentimento dos adolescentes e com o consentimento dos pais. Posteriormente transcritas, sendo realizado a análise de conteúdo temático que seguiu 3 fases: a primeira préanálise foi realizada leitura exaustiva dos dados, seguida da organização do material e formulação de hipóteses. $\mathrm{Na}$ sequência, foi realizada a exploração do material, ou seja, a codificação dos dados brutos. Na terceira e última fase, os dados foram interpretados e paralelamente delimitados os eixos temáticos, a partir da compreensão dos significados estabelecidos (Bardin, 2011).

Para manter o anonimato dos participantes, as falas foram identificadas, no texto por pseudônimos retirados da sala de bate - papo do site Universo Online (UOL). Os aspetos éticos foram respeitados de acordo com as recomendações da Resolução do Conselho Nacional de Saúde $n^{\circ} 466 / 2012$ que prescreve a ética em pesquisa com seres humano (Ministério da Saúde,2012). Sendo o projeto de pesquisa aprovado pelo Comitê de Ética em Pesquisa da instituição proponente e coparticipante sob o no 692.112 . 


\section{RESULTADOS}

Foram participantes dessa pesquisa 09 meninas e 05 meninos, com idade de 14 a 20 anos. Após a análise dos dados obtidos, o estudo apontou os aspetos positivos e negativos relacionado a busca de informações sobre doença crônica por adolescentes nas redes virtuais.

\section{Aspetos Positivos Apresentados pelos Adolescentes}

O esclarecimento quanto ao processo da doença, provenientes da obtenção de informação por meio virtual, foi um aspeto muito significativo para os adolescentes como se pode perceber no relado abaixo:

"Me ajudou a aceitar, porque eu não aceitava aí eu aceitei. Quando eu aceitei eu melhorei, teve uma época que eu não estava aceitando de jeito nenhum, aí minha mãe falou ou eu aceitava ou eu aceitava. Aí eu tive que aceitar né?. Aí eu comecei a procurar saber mais, aí eu descobri como é que é, que essa doença não é TÃO ruim como eu pensei que ela era, é só cuidar" [Mily].

As informações obtidas na internet pela adolescente contribuíram para que a mesma aceitasse sua condição, e consequentemente, despertou o interesse de obter mais conhecimento a fim de se sentir bem e melhor. Esse processo de conhecer e aceitar a doença crônica desperta nas adolescentes potencialidades de enfrentamento e possibilidades de serem felizes.

Além disso, o esclarecimento associado aos relatos de experiência de outras pessoas com o mesmo diagnóstico, contribuiu como incentivo ao tratamento, conforme o depoimento a seguir:

"Eu vi que tinha muita gente com o mesmo problema ..., É ...tinha gente se tratando, tinha gente falando que se não tratar ...que deixou de tratar quando jovem aí quando ficou adulto ficou pior, fica difícil de se tratar, perde o controle. Aí, tipo, me deu um incentivo para eu continuar me tratando..." [Aventureiro].

Outra adolescente relatou como a informação na internet a ajudou a compreender melhor o procedimento pelo qual ela havia passado.

"Eles falaram que colocaram um catéter duplo "J" em mim, e aí eu queria saber o quê que era. Aí quando eu cheguei em casa, aí eu fui pesquisar e vê o que era e eu vi.[...] aí eu já entendi , eu vi como era, que era um.. um ligado no rim, um ligado na bexiga. Dá pra entender, não cheguei a ler, nem entrar no site, mas vi imagens [Butterfly].

Conhecer a doença que possui é uma necessidade, e as redes virtuais tem auxiliado os adolescentes ao autocuidado e a autonomia:
"Tem que diminuir o sal. Tem que evitar comer algumas coisas. Beber bastante água, essas coisas" [Pocahontas S2].

“Tomar bastante água, comer menos sal, e tomar o remédio direitinho" [Penélope Charmosa].

\section{Aspetos Negativos Apontados pelos Adolescentes}

Ao recorrer à internet para obtenção de informação, além do sucesso esperado, ele pode se deparar com um universo científico rigoroso que impossibilitará o êxito quanto ao entendimento sobre o conteúdo buscado devido a sua imaturidade.

É que tem coisas que eram muito científicas...a linguagem, não deu pra entender muito não.[Kalado 45].

Além do termo "coisas científicas", o vocabulário técnico da área da saúde foi descrito por uma adolescente como "nomes esquisitos".

Eu pesquisei lá, o que era esse negócio, só que aí tem vários nomes esquisitos. Aí eu...aí eu nunca mais pesquisei não [Princesa].

Ao serem questionados sobre como eles identificam que a veracidade das informações e como eles sabem que o site é seguro, alguns adolescentes não souberam responder, outros se basearam em sua experiência, seus sintomas:

Porque às vezes, eles estavam falando do que eu já tinha passado, é... dores nas articulações, às vezes apareciam uma pintinhas vermelhas, no meu caso apareceu, aí eu sabia que tinha fundamento porque algumas situações eu já vivi com o lúpus [Saori].

Outra possibilidade para identificar a veracidade do conteúdo foi por estar disponível em sites populares, como o Wikipédia, por exemplo.

Verídico? Porque foi a wikipédia! Então vem assim, poxa foi a wikipédia, a wikipédia mente, mas nem tanto quanto os outros sites, então assim, minha primeira informação foi a wikipédia, aí a partir daí, da wikipédia, eu peguei a wikipédia como fonte para todas as outras pesquisas e muita coisa que eu vi na wikipédia batia com o que eu tava passando no hospital, então foi isso, eu nunca cheguei em outro site, fui e procurei, geralmente foi na wikipédia, só, então foi bem assim [Internauta]. 


\section{DISCUSSÃO}

As redes sociais virtuais criadas nas mídias virtuais, têm-se tornado um forte instrumento adotado com mais frequência para promoção de apoio, incentivo, troca de experiências entre outras atividades, ou seja, ocorre um rompimento das fronteiras com o auxílio das tecnologias. Para Snyder et al. (2011), as ferramentas de comunicação da Internet, tais como salas de bate-papo, fóruns de discussão, blogs e grupos de apoio online podem ajudar os pacientes a compartilhar experiências e fazer perguntas através do apoio mútuo dos outros sobreviventes da condição crônica.

$\mathrm{O}$ apoio para o tratamento pode vir de pessoas em situações semelhantes com insuficiência similar (Dumaji \& Tijsse, 2011). Assim surge um sentimento de pertença, quando encontram grupos que vivem a mesma situação, o mesmo problema de saúde, um espaço que é deles, onde podem inclusive formar vínculos sociais. Neste sentido, Moreira \& Souza (2002) refletem esses espaços como "identidades comuns", onde se identificam como os iguais e discutem a formação de redes de apoio social, pois na adolescência é essencial para $o$ adolescente pertencer a um grupo e sentir-se aceite. Também foi possível perceber nas falas dos adolescentes que o uso das mídias os ajudaram a compreender o processo da doença e intervenções realizadas. O conhecimento que o adolescente possui sobre a doença será determinante no desenvolvimento de estratégias positivas para o enfrentamento, as quais minimizarão os estressores que advirão na sua vida como doente crônico (Araújo, Collet, Gomes \& Amador, 2011a).

Informações de saúde de alta qualidade podem ser fornecidas através de sites, fóruns, blogs e redes sociais, que têm sido alguns dos canais mais populares para a promoção da saúde entre os jovens. Nos últimos 10 anos, na França, por exemplo, três de quatro pessoas têm acesso à internet e o uso da internet é maior entre os jovens que os adultos (Beck, Lopes, Giuliano \& Borgatto, 2011).

A comunicação é um processo complexo, subjetivo e que abarca a perceção, a compreensão e a transmissão de mensagens entre pacientes e profissionais de saúde, de modo verbal e não-verbal. Entre o modo não-verbal, menciona-se a busca de conteúdos nas mídias virtuais (Tamaki, Meneguin, Alencar \& Luppi, 2014).

Em relação aos saberes dos adolescentes, quanto mais ciência $\mathrm{o}$ adolescente tiver sobre as causas que o afetam terá mais probabilidade de ser ativo e autônomo diante de sua própria vida (Araújo et al. 2011a).
A partir do diagnóstico de uma doença crônica o adolescente necessita se adequar a um novo estilo de vida, constituído de consultas médicas, realização de exames, uso de medicamentos, limitações físicas, restrições alimentares, sinais e sintomas da doença e uma série de alterações no seu cotidiano, inclusive possíveis internações (Almeida, Lopes \& Simões, 2014).

$\mathrm{O}$ adolescente que convive com uma doença crônica precisa construir autonomia para o cuidado de si, e não ficar dependente do cuidado da família, o que pode levá-lo a se sentir incapaz de enfrentar circunstâncias cotidianas sozinhos (Araújo, Collet, Gomes e Nóbrega, 2011b). Com isso, podemos considerar a busca por informações na internet pelo adolescente, a compreensão do diagnóstico e a forma de cuidado da saúde possibilitam uma independência, incluindo o próprio adolescente no tratamento como um participante ativo, proporcionando o vir a sentir-se bem, conforme foi expresso por eles.

"Navegar" pelo ambiente virtual pode ser uma tarefa simples, mas nem sempre é fácil como se espera, ainda mais para o mundo do adolescente, quando uma série de questionamentos perturba sua mente sobre outro mundo desconhecido, que é o da doença crônica.

Desde a chegada de intervenções de mídia social para fins relacionados com a saúde, tornou-se claro que nem todas estas intervenções são realmente bem-sucedidas (Van de Belt \& Engelen, 2013).

Deve-se atentar para a forma como é disponibilizado o conteúdo na internet, se de fato pretende-se que os efeitos sejam informativos, é oportuno considerar a diversidade populacional com as doenças crônicas, e não as generalizar.

A informação é fundamental para o cuidado centrado no paciente, e na área da informática em saúde tem acontecido uma evolução nos últimos anos pautada em como a informação é adquirida, armazenada e utilizada nos cuidados de saúde. Talvez o maior desafio para pacientes e provedores seja identificar a informação que é de alta qualidade e que aumente (e não impeça) suas interações. Se há desinformação, os efeitos nos pacientes podem envolver a ansiedade ou questionamentos a respeito da competência médica (Snyder et al., 2011). Ao inserir uma informação em um meio virtual, necessita-se atentar para o público que se pretende alcançar e os possíveis públicos que poderão acessá-los, pois um conteúdo lançado na rede precisa alcançar o seu objetivo proposto e apresentar segurança para quem irá adquiri-lo. 
Já que os adolescentes, pela sua imaturidade, e por estarem em processo de descobertas apresentarão essa dificuldade para assimilação e distinção entre o correto e o errado.

No Brasil, como forma de qualificar as informações de saúde que são disponibilizadas na internet, destacamse iniciativas como a da Fiocruz, com seu Laboratório denominado Laiss (Laboratório Internet, Saúde e Sociedade), que tem o intuito de criar mecanismos capazes de avaliar a confiabilidade de sites médicos e de informações de saúde veiculadas na rede; e também as ações do Conselho Regional de Medicina do Estado de São Paulo (CREMESP), que criou, em 20 de fevereiro de 2001, uma resolução (n. ${ }^{\text {9 } 97) ~ q u e ~ d i s p o ̃ e ~ s o b r e ~ a ~ i d e-~}$ alização, criação, manutenção e atuação profissional em sites, páginas ou portais sobre medicina e saúde na internet (Moretti, Oliveira \& Silva, 2012).

Os jovens têm dificuldades de julgar a qualidade do conteúdo na internet, que esteja relacionado à saúde. Para isso, é fundamental ajudar os jovens a encontrar e utilizarem as informações em saúde online mais válidas. Uma estratégia vem a ser a criação de sites referenciados, como já ocorre nos Estados Unidos (Beck et al., 2011). Torna-se necessário o incentivo a autorregulação do setor para se estabelecerem padrões mínimos de qualidade, segurança e confiabilidade dos sites de saúde. A resolução (N. ${ }^{\circ}$ 97) ainda sinaliza que o usuário da internet, na busca de informações, serviços ou produtos de saúde online, tem o direito de exigir das organizações e indivíduos responsáveis pelos sites: Transparência; Honestidade; Qualidade; Consentimento Livre e Esclarecido; Privacidade; Ética Médica e Responsabilidade e Procedência (Moretti et al., 2012).

Esses aspetos negativos apresentados como, por exemplo - a dificuldade de reconhecer um site como seguro ou verdadeiro, que apresente credibilidade para quem utiliza seu conteúdo merecem bastante atenção, por se tratar de conteúdos voltados para a saúde, os prejuízos podem ser imensuráveis.

Então, ainda que a internet seja vista como um todo, precisa-se lembrar que por detrás desses ícones virtuais existem pessoas, seres humanos, ainda que capacitados ou não para o exercício dessa atividade online, essas pessoas são passíveis de erros, acertos e todas as potencialidades humanas. $\mathrm{Na}$ ida até as redes virtuais para obtenção de informação a fim de compreender o processo de seu diagnóstico espera-se que a resposta venha de um profissional da saúde, especialmente na figura do enfermeiro, pois o mesmo é visto como um profissional da educação em saúde e durante os depoimentos dos adolescentes, a imagem do enfermeiro passou desapercebida.

\section{CONCLUSÃO}

Embora seja complexo o processo de adaptação do adolescente à sua condição crônica, é de suma importância à participação do adolescente no seu processo de recuperação, uma vez que entre os benefícios está o autocuidado, a adesão ao tratamento e a melhora na autoestima. A busca por informações na internet pelos adolescentes, a compreensão do diagnóstico e a forma de cuidado da saúde possibilitam uma independência, incluindo o próprio adolescente no tratamento como um participante ativo.

A busca por informações em saúde obtidas no ambiente virtual caracterizou-se como uma estratégia dos adolescentes deste estudo a enfrentar a doença crônica e manter o tratamento. Por outro lado, não podemos desviar o olhar dos aspetos negativos que dificultaram a compreensão da informação. Entre eles temos a linguagem científica, o excesso ou falta de conteúdo, e a dificuldade para identificação de sites seguros, com informações confiáveis.

Embora alguns órgãos venham se debruçando sobre as maneiras como as informações são lançadas no meio virtual, isso ocorre ainda de forma pouco expressiva. Por isso, é necessária agilidade no estabelecimento de critérios que devem ser adotados pelos organizadores de sites, a fim de divulgar seu conteúdo de maneira segura e com credibilidade.

O crescimento das mídias sociais ocorre expressivamente, possibilitando a existência de um espaço a ser valorizado pelo enfermeiro. Uma vez que os enfermeiros se inseriram neste mundo virtual, eles devem proteger a privacidade do paciente, manter a confidencialidade de suas ações no exercício do cuidado, e compreender a importância de sua presença nesta nova realidade, pois a disponibilidade de conteúdos deturpados é um risco ao bem-estar do paciente.

\section{IMPLICAÇÕES PARA A PRÁTICA CLÍNICA}

O crescimento das mídias sociais ocorre expressivamente, possibilitando a existência de um espaço a ser valorizado pelo enfermeiro. Uma vez que os enfermeiros ao serem inseridos neste mundo virtual, eles devem proteger a privacidade do paciente, manter a confidencialidade de suas ações no exercício do cuidado, e compreender a importância de sua presença nesta nova realidade, pois a disponibilidade de conteúdos deturpados é um risco ao bem-estar do paciente. 


\section{REFERÊNCIAS BIBLIOGRÁFICAS}

Almeida, I. S., Lopes, L. M. S., \& Simões, S. M. F. (2014). Ser adolescente vivenciando a situação crônica de Saúde: Uma compreensão Heideggeriana. Ciência, Cuidado e Saúde, 11(4), 704-711. doi: dx.doi.org/10.4025/ cienccuidsaude.v11i4.13214

Araújo, Y. B., Collet, N., Gomes, I. P., \& Amador, D. D. (2011a). Saberes e experiências de adolescentes hospitalizados com doença crônica. Revista de Enfermagem da Universidade do Estado do Rio de Janeiro, 19(2), 274-279.

Araújo, Y. B., Collet, N., Gomes, I. P., \& Nóbrega, R. D. (2011b). Enfrentamento do adolescente em condição crônica: Importância da rede social. Revista Brasileira de Enfermagem, 64(2), 281-286.

Bardin, L. (2011). Análise de conteúdo. Lisboa: Edições 70 .

Beck, C. C., Lopes, A. S., Giuliano, I. C. B., \& Borgatto, A. F. (2011). Fatores de risco cardiovascular em adolescentes de município do sul do Brasil: Prevalência e associações com variáveis demográficas. Revista Brasileira de Epidemiologia, 14(1), 36-49.

Dumaij, A. C. M., \& Tijsse, E. C. G. (2011). On-line health companion contact among chronically ill in the Netherlands. Health and Technology, 1(1), 5-23.

Greene, J. A., Choudhry, N. K., Kilabuk, E., \& Shrank, W. H. (2011). Online social networking by patients with diabetes: A qualitative evaluation of communication with Facebook. Journal of General Internal Medicine, 26(3), 287-292.

Ministério da Saúde. Conselho Nacional de Saúde. (2012). Diretrizes e normas regulamentadoras de pesquisa envolvendo seres humanos. Resolução n. 466, de 12 de dezembro de 2012. Brasília.
Moreira, M. C. N., \& Souza, W. S. (2002). A microssociologia de Erving Goffman e a análise relacional: Um diálogo metodológico pela perspectiva das redes sociais na área de saúde. Teoria e Sociedade, 9, 38-61.

Moretti, F. A., Oliveira, V. E., \& Silva, E. M. K. (2012). Acesso a informações de saúde na internet: Uma questão de saúde pública? Revista da Associação Médica Brasileira, 58(6), 650-658.

Morgado, T., \& Botelho, M. R. (2014). Implicações promotoras da literacia em saúde mental dos adolescentes: Uma revisão sistemática da literatura. Revista Especial da Sociedade Portuguesa de Enfermagem de Saúde Mental (Ed. Esp. 1), 90-96.

Santos, G. S., Tavares, C. M. M., Ferreira, R. E., \& Pereira, C. S. F. (2015). Rede social e virtual de apoio ao adolescente que convive com doença crônica: Uma revisão integrativa. Aquichan, 15(1), 60-74. doi: dx.doi. org/10.5294/aqui.2015.15.1.7

Snyder, C. F., Wu, A. W., Miler, R. S., Jensen, R. E., Bantug, E. T., \& Wolff, A. C. (2011). The role of informatics in promoting patient-centered care. The Cancer Journal, 17(4), 211-218.

Tamaki, C. M., Meneguin, S., Alencar, R.A., \& Luppi, C. H. B. (2014). Care to terminal patients. Perception of nurses from the intensive care unit of a hospital. Investigación y Educación en Enfermería, 32(3), 414-420.

Van de Belt, T. H., Engelen, L. J. L. P. G. (2013). Internet and social media for health-related information and communication in health care: Preferences of the Dutch general population. Journal of Medical Internet Research, 15(10), e220.

Velden, M. V., \& Khaled, E. E. (2013). "Not all my friends need to know": A qualitative study of teenage patients, privacy, and social media. Journal of the American Medical Informatics Association, 20(4), 16-24. 